\title{
1-Y-E3-3 YIA
}

Development of a novel epitope mapping method (REMAP) using RIEDL tag system

Asano Teizo, Mika Kaneko, Yukinari Kato

Dept. Antibody Drug Development, Tohoku University Grad. Sch. of Med. 